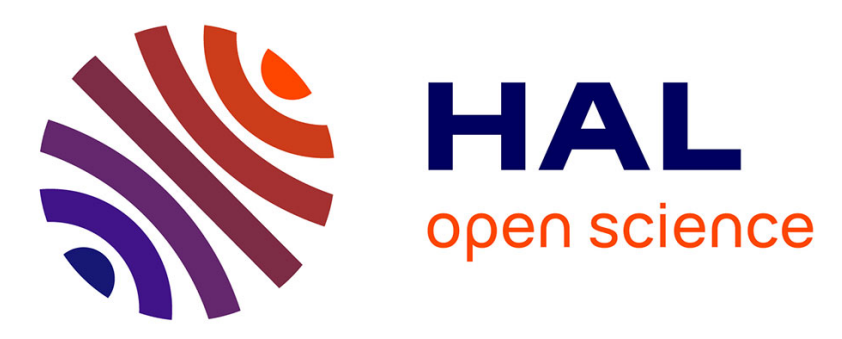

\title{
Statistical Abstraction Boosts Design and Test Efficiency of Evolving Critical Systems
}

\author{
Axel Legay, Sean Sedwards
}

\section{To cite this version:}

Axel Legay, Sean Sedwards. Statistical Abstraction Boosts Design and Test Efficiency of Evolving Critical Systems. Leveraging Applications of Formal Methods, Verification and Validation. Technologies for Mastering Change, EasyConference, Oct 2014, Corfu, Greece. pp.4 - 25, 10.1007/978-3-66245234-9_2. hal-01087858

\section{HAL Id: hal-01087858 \\ https://inria.hal.science/hal-01087858}

Submitted on 26 Nov 2014

HAL is a multi-disciplinary open access archive for the deposit and dissemination of scientific research documents, whether they are published or not. The documents may come from teaching and research institutions in France or abroad, or from public or private research centers.
L'archive ouverte pluridisciplinaire HAL, est destinée au dépôt et à la diffusion de documents scientifiques de niveau recherche, publiés ou non, émanant des établissements d'enseignement et de recherche français ou étrangers, des laboratoires publics ou privés. 


\title{
Statistical Abstraction Boosts Design and Test Efficiency of Evolving Critical Systems
}

\author{
Axel Legay and Sean Sedwards \\ Inria Rennes - Bretagne Atlantique
}

\begin{abstract}
Monte Carlo simulations may be used to efficiently estimate critical properties of complex evolving systems but are nevertheless computationally intensive. Hence, when only part of a system is new or modified it seems wasteful to re-simulate the parts that have not changed. It also seems unnecessary to perform many simulations of parts of a system whose behaviour does not vary significantly.

To increase the efficiency of designing and testing complex evolving systems we present simulation techniques to allow such a system to be verified against behaviour-preserving statistical abstractions of its environment. We propose a frequency domain metric to judge the a priori performance of an abstraction and provide an a posteriori indicator to aid construction of abstractions optimised for critical properties.
\end{abstract}

\section{Introduction}

The low cost of hardware and demand for increased functionality make modern computational systems highly complex. At the same time, such systems are designed to be extensible and adaptable, to account for new functionality, increased use and new technology. It is usually cost-efficient to allow a system to evolve piece-wise, rather than replace it entirely, such that over time it may not respect its original specification. A key challenge is therefore to ensure that the critical performance of evolving systems is maintained up to the point of their obsolescence.

The basic challenge has been addressed by robust tools and techniques developed in the field of software engineering, which allow designers to specify and verify the performance of complex systems in terms of data flow. The level of abstraction of these techniques often does not include the precise dynamical behaviour of the implementation, which may critically affect performance. Hence, to guarantee that the implementation of a system respects its specification it is necessary to consider detailed dynamical models. In particular, it is necessary to consider dynamics that specifically model the uncertainty encountered in real deployment.

\subsection{Our Approach}

To address the problem of designing and testing evolving critical systems [16] we focus on efficient ways to construct and formally verify large dynamical models 
whose structure evolves over time. In particular, we consider systems comprising components whose dynamics may be represented by continuous time Markov chains (CTMC). CTMCs model uncertainty by probabilistic distributions and may also include deterministic behaviour (i.e., that happens with probability 1 in a given state). Importantly, CTMCs allow the verification and quantification of properties that include real time.

We consider verification of dynamical properties using model checking, where properties are specified in temporal logic [6]. Importantly, such logics typically include an until operator, which expresses properties that include temporal causality. To quantify uncertainty and to consider events in real time, numerical model checking extends this idea to probabilistic systems, such as modelled by CTMCs. Current numerical model checking algorithms are polynomial in the size of the state space $[1,6]$, but the state space scales exponentially with respect to the number of interacting variables, i.e., the intuitive notion of the size of the system. Techniques such as symbolic model checking [5], partial order reduction [14], bounded model checking [3] and abstraction refinement [7] have made model checking much more efficient in certain cases, but the majority of real systems remain intractable.

Statistical model checking (SMC) is a Monte Carlo technique that avoids the 'state explosion problem' [6] of numerical model checking by estimating the probability of a property from the proportion of simulation traces that individually satisfy it. SMC is largely immune to the size of the state space and takes advantage of Bernoulli random variable theory to provide confidence bounds for its estimates $[20,25]$. Since SMC requires independent simulation runs, verification can be efficiently divided on parallel computing architectures. SMC can thus offer a tractable approximate solution to industrial-scale numerical model checking problems that arise during design or certification of a system. SMC may nevertheless be computationally expensive with rare properties (which are often critical) and when high precision is required.

SMC relies on simulation, so in this work we propose a technique of statistical abstraction to boost the efficiency of simulation. We show how to construct adequate statistical abstractions of external systems and we provide a corresponding stochastic simulation algorithm that maintains existing optimisations and respects the causality of the original system. We demonstrate that it is possible to make useful gains in performance with suitable systems. We provide a metric to judge a priori that an abstraction is good and an indicator to warn when the abstraction is not good in practice. Importantly, we show that a statistical abstraction may be optimised for critical rare properties, such that overall performance is better than simulation without abstraction.

The basic assumption of our approach is that we wish to apply SMC to a system comprising a core system in an environment of non-trivial external systems. In particular, we assume that properties of the external systems are not of interest, but that the environment nevertheless influences and provides input to the core. In this context, we define an external system to be one whose behaviour is not affected by the behaviour of the core or other external systems. This may 
seem like a severe restriction, but this topology occurs frequently because it is an efficient and reliable way to construct large systems from modules. A simple example is a network of sensing devices feeding a central controller. Our case study is a complex biological signalling network that has this topology. To consider more general interactions would eliminate the modularity that makes statistical abstraction feasible.

Our idea is to replace the external systems with simple characterisations of their output, i.e., of the input to the core system. To guarantee the correct time-dependent behaviour of the environment, we find the best approach is to construct an abstraction based on an empirical distribution of traces, created by independent simulations of the external systems. During subsequent investigation, the core system is simulated against traces chosen uniformly at random from the empirical distributions of each of the external systems. The abstractions are constructed according to a metric based on frequency domain analysis. We make gains in performance because $(i)$ the external systems are simulated in the absence of other parts of the system, $(i i)$ the empirical distributions contain only the transitions of the variables that affect the core and (iii) the empirical distributions contain the minimum number of traces to adequately represent the observed behaviour of the variables of interest.

SMC generally has very low memory requirements, so it is possible to take advantage of the unused memory to store distributions of pre-simulated traces. Such distributions can be memory intensive, so we also consider memory-efficient abstractions using Gaussian processes, to approximates the external system on the fly. We give results that demonstrate the potential of this approach, using frequency domain analysis to show that it is possible to construct abstractions that are statistically indistinguishable from empirical abstractions.

Although our idea is simple in concept, we must show that we can achieve a gain in performance and that our algorithm produces correct behaviour. The first challenge arises because simulation algorithms are optimised and SMC already scales efficiently with respect to system size. We must not be forced to use inefficient simulation algorithms and the cost of creating the abstractions must not exceed the cost of just simulating the external systems. The second challenge arises because, to address the first challenge, we must adapt and interleave the simulation algorithms that are most efficient for each part of the system.

\subsection{Related Work}

Various non-statistical abstractions have been proposed to simplify the formal analysis of complex systems. These include partial order reduction [14], abstract interpretation [8] and lumping [19]. In this work we assume that such behaviourpreserving simplifications have already been applied and that what remains is a system intractable to numerical techniques. Such systems form the majority.

Approximating the dynamical behaviour of complex systems with statistical processes is well established in fields such as econometrics and machine learning. Using ideas from these fields, we show in Section 4.2 that abstracting external systems as Gaussian processes may be a plausible approach for SMC. We do 
not attempt to survey the considerable literature on this subject here, but mention some recent work [4] that, in common with our own, links continuous time Markov chains, temporal logic and Gaussian processes. The authors of [4] address a different problem, however. They use a Gaussian process to parametrise a CTMC that is not fully specified, according to temporal logic constraints.

Of greatest relevance to our approach is that of [2], which considers a complex heterogeneous communication system (HCS) comprising multiple peripheral devices (sensors, switches, cameras, audio devices, displays, etc.), that communicate bidirectionally via a central server. The authors of [2] use SMC to verify the correct communication timing of the HCS. To increase efficiency they replace the peripherals with static empirical distributions of their respective communication timings, generated by simulating the entire system. In contrast to our approach, $(i)$ the quality of the statistical abstractions is not specified or measured, (ii) the distributions of the statistical abstractions are static (not varying with time) and ( $i i i)$ the statistical abstractions are generated by simulating the entire system. The consequence of $(i)$ is that it is not possible to say whether the abstractions adequately encapsulate the behaviour of the peripherals. The consequence of $(i i)$ is that the abstractions do not allow for different behaviour at different times: a sequence of samples from the abstraction does not in general represent samples of typical behaviour, hence the abstraction does not preserve the behaviour of the original system. The consequence of (iii) is that the abstractions are generated at the cost of simulating the entire system, thus only allowing significant gains with multiple queries. A further consequence of $(i i)$, in common with our own approach, is that the approach of [2] cannot model bidirectional communication. This remains an open problem.

\subsection{Structure of the Paper}

In Section 2 we use the equivalence of classic stochastic simulation algorithms to show how the simulation of a complex system may be correctly decomposed. We then present the compositional stochastic simulation algorithm we use for abstraction. In Section 3 we motivate the use of empirical distributions as abstractions and show how they may be validated using frequency domain analysis. In Section 3.2 we provide a metric to judge the a priori quality of an abstraction and in Section 3.3 we provide an a posteriori metric to help improve the critical performance of an abstraction. In Section 4 we give a brief overview of our biological case study and present results using empirical abstractions. In Section 4.2 we present promising results using Gaussian process abstractions. We conclude with Section 5. Appendix A gives full technical details of our case study.

\section{Stochastic Simulation Algorithms}

We consider systems generated by the parallel composition of stochastic guarded commands over state variables. A state of the system is an assignment of values to the state variables. A stochastic guarded command (referred to simply as a 
command) is a guarded command [9] with a stochastic rate, having the form (guard, action, rate). The guard is a logical predicate over the state, enabling the command; the rate is a function of the state, returning a positive real-valued propensity; the action is a function that assigns new values to the state variables. The semantics of an individual command is a continuous time Markov jump process, with an average stochastic rate of making jumps (transitions from one state to another) equal to its propensity. The semantics of a parallel composition of commands is a Markov jump process where commands compete to execute their actions. An evolution of the system proceeds from an initial state at time zero to a sequence of new states at monotonically increasing times, until some time bound or halting state is reached. The time between states is referred to as the delay. A halting state is one in which no guard is enabled or in which the propensities of all enabled commands are zero. Since the effect is equivalent, in what follows we assume, without loss of generality, that a disabled command has zero propensity.

In the following subsections we use the equivalence of classic stochastic simulation algorithms to formulate our compositional simulation algorithm.

\subsection{Direct Method}

Each simulation step comprises randomly choosing a command according to its probability and then independently sampling from an exponential distribution to find the delay. Given a system containing $n$ commands whose propensities in a state are $p_{1}, \ldots, p_{n}$, the probability of choosing command $i$ is $p_{i} / \sum_{j=1}^{n} p_{j}$. Command $\nu$ is thus chosen by finding the minimum value of $\nu$ that satisfies

$$
\mathcal{U}\left(0, \sum_{j=1}^{n} p_{j}\right) \leq \sum_{i=1}^{\nu} p_{i} .
$$

$\mathcal{U}\left(0, \sum_{j=1}^{n} p_{j}\right)$ denotes a value drawn uniformly at random from the interval $\left(0, \sum_{j=1}^{n} p_{j}\right)$. The delay time is found by sampling from an exponential probability density with amplitude equal to the sum of the propensities of the competing commands. Hence,

$$
t=\frac{-\ln (\mathcal{U}(0,1])}{\sum_{j=1}^{n} p_{j}} .
$$

$\mathcal{U}(0,1]$ denotes a value drawn uniformly at random from the interval $(0,1]$.

\section{$2.2 \quad$ First Reaction Method}

In each visited state a 'tentative' delay time is generated for every command, by randomly sampling from an exponential distribution having probability density function $p_{i} e^{-p_{i} t}$, where $p_{i}$ is the propensity of command $i$. The concrete delay time of this step is set to the smallest tentative time and the action to execute belongs to the corresponding command. Explicitly, the tentative delay time of command $i$ is given by $t_{i}=-\ln \left(\mathcal{U}_{i}(0,1]\right) / p_{i} \cdot \mathcal{U}_{i}(0,1]$ denotes sample $i$ drawn 
uniformly at random from the interval $(0,1]$. Commands with zero propensity are assigned infinite times. It is known that the FRM is equivalent to the DM [12], but for completeness we give a simple proof.

Let $P_{i}=p_{i} e^{-p_{i} t}$ be the probability density of the tentative time $t_{i}$ of command $i$. Since the tentative times are statistically independent, the joint probability density of all tentative times is simply the product of the $n$ individual densities with respect to $n$ time variables. The marginal density of tentative time $t_{i}$ when it is the minimum is given by

$$
\begin{aligned}
P_{i}^{\min } & =\int_{t_{i}}^{\infty} \mathrm{d} t_{1} \cdots \int_{t_{i}}^{\infty} \mathrm{d} t_{i-1} \int_{t_{i}}^{\infty} \mathrm{d} t_{i+1} \cdots \int_{t_{i}}^{\infty} \mathrm{d} t_{n} P_{1} \cdots P_{n} \\
& =p_{i} e^{-t \sum_{j=1}^{n} p_{j}} .
\end{aligned}
$$

Since only one command can have the minimum tentative time (the probability of two samples having the same value is zero), the overall density of times of the FRM is the sum of the marginal densities

$$
\sum_{i=1}^{n} P_{i}^{\min }=\sum_{j=1}^{n} p_{j} e^{-t \sum_{k=1}^{n} p_{k}} .
$$

This is the same density used by the DM (2).

\subsection{Simulating Subsystems}

A system described by a parallel composition of commands may be decomposed into a disjoint union of subsets of commands, which we call subsystems to be precise. By virtue of the properties of minimum and the equivalence of the DM and FRM, in what follows we reason that it is possible to simulate a system by interleaving the simulation steps of its subsystems, allowing each subsystem to be simulated using the most efficient algorithm.

Using the FRM, if we generate tentative times for all the commands in each subsystem and thus find the minimum tentative time for each, then the minimum of such times is also the minimum of the system considered as a whole. This time corresponds to the command whose action we must execute. By the equivalence of the DM and FRM, we can also generate the minimum tentative times of subsystems using (2) applied to the subset of propensities in each subsystem. Having chosen the subsystem with the minimum tentative time, thus also defining the concrete delay time, the action to execute is found by applying (1) to the propensities of the chosen subsystem. Similarly, we may select the subsystem by applying (1) to combined propensities $q_{1}, \ldots, q_{i}, \ldots$, where each $q_{i}$ is the sum of the propensities in subsystem $i$. Having selected the subsystem, we can advance the state of the whole system by applying either the DM or FRM (or any other equivalent algorithm) to just the subsystem.

We define an external subsystem to be a subsystem whose subset of state variables are not modified by any other subsystem. We define a core subsystem to be a subsystem that does not modify the variables of any other subsystem. 
Given a system that may be decomposed into a core subsystem and external subsystems, it is clear that traces of the external subsystems may be generated independently and then interleaved with simulations of the core subsystem. Simulations of the core subsystem, however, are dependent on the modifications to its state variables made by the external subsystems.

By virtue of the memoryless property of exponential distributions, the FRM will produce equivalent results using absolute times instead of relative delay times. This is the basis of the 'next reaction method' (NRM, [10]). Precisely, if a command is unaffected by the executed action, its tentative absolute time may be carried forward to the next step by subtracting the absolute tentative time of the selected command. Intuitively, the actions of commands that are momentarily independent are interleaved with the actions of the other commands. Since, by assumption, no action of the core or any other subsystem may affect the commands of the external subsystems, it is correct to simulate the core subsystem in conjunction with the interleaved absolute times of events in the simulations of the external subsystems. Moreover, it is only necessary to include transitions in the abstractions that modify the propensities of the commands in the core.

\subsection{Compositional Stochastic Simulation Algorithm}

Given a system comprising a core subsystem and external subsystems, the pseudocode of our compositional simulation algorithm is given in Algorithm 1. The basic notion is intuitive: at each step the algorithm chooses the event that happens next. To account for the fact that the core is not independent of the external subsystems, the algorithm "backtracks" if the simulated time of the core exceeds the minimum next state time of the external subsystems.

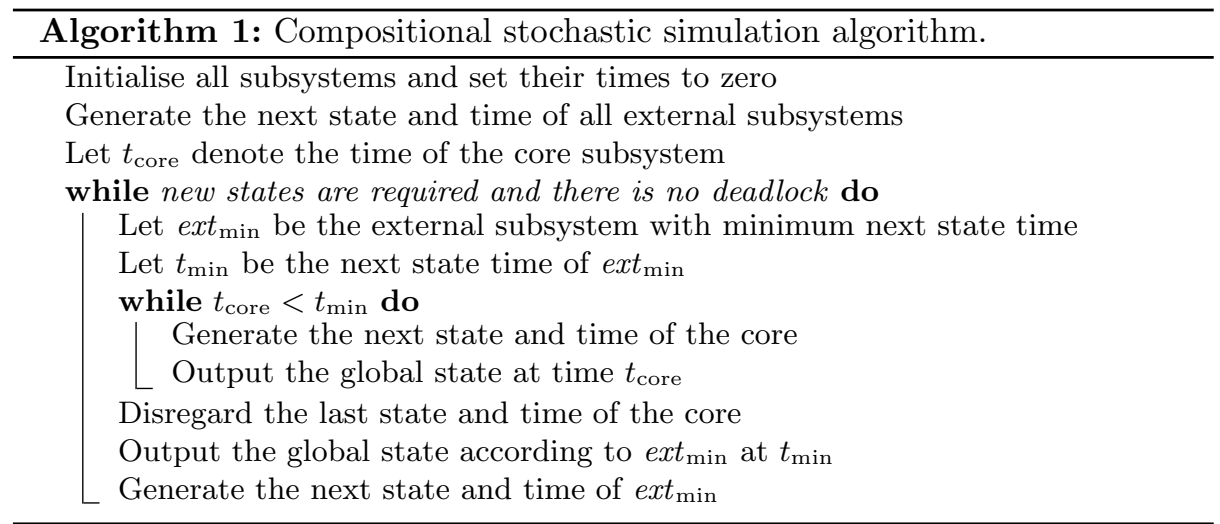

Algorithm 1 does not specify how the next states of each subsystem will be generated. Importantly, we have shown that it is correct to simulate external subsystems independently, so long as the chosen simulation algorithms produce 
traces equivalent to those of the FRM and DM. Algorithm 1 thus provides the flexibility to use the best method to simulate each part of the system. With a free choice of algorithm, worst case performance for an arbitrary subsystem of $n$ commands is $\mathcal{O}(n)$ per step. If a subsystem has low update dependence between commands, asymptotic performance could be as low as $\mathcal{O}\left(\log _{2} n\right)$ using the NRM [10].

Algorithm 1 is our abstraction simulation algorithm, using statistical abstractions to provide the next states of the external subsystems. In the case of empirical distribution abstractions, this amounts to reading the next state of a randomly selected stored trace. In the case of Gaussian process abstractions, new states need only be generated when old states are consumed.

\section{Empirical Distribution Abstraction}

We propose the use of a relatively small number of stored simulation traces as an empirical distribution abstraction of an external subsystem, where the traces need only contain the changes of the output variables.

The output trace of a stochastic simulation is a sequence of states, each labelled with a monotonically increasing time. The width of the trace is equal to the number of state variables, plus one for time. Each new state in the full trace corresponds to the execution of one of the commands in the model. Typically, the action of a single command updates only a small subset of the state variables. Hence, the value of any variable in the trace is likely to remain constant for several steps. Given that the core system is only influenced by a subset of the variables in the external system, it is possible to reduce the width of the abstraction by ignoring the irrelevant variables. Moreover, it is possible to reduce the length of the trace by ignoring the steps that make no change to the output variables.

We argue that we may adequately approximate an external subsystem by a finite number of stored traces, so long as their distribution adequately "covers" the variance of behaviour produced by the subsystem. To ensure that a priori the empirical distribution encapsulates the majority of typical behaviour, in Section 3.2 we provide a metric based on frequency domain analysis. Recognising that some (rare) properties may be critically dependent on the external system (i.e., depend on properties that are rare in the empirical distribution of the external subsystem), in Section 3.3 we provide an indicator to alert the user to improve the abstraction. The user may then create abstractions that favour rare (critical) properties and perform better than standard simulation.

\subsection{Frequency Domain Analysis}

Characterising the "average" empirical behaviour of stochastic systems is challenging. In particular, the mean of a set of simulation traces is not adequate because random phase shifts between traces cause information to be lost. Since delay times between transitions are drawn from exponential random variables, 
two simulations starting from the same initial state may drift out of temporal synchronisation, even if their sequences of transitions are identical. For example, in the case of an oscillatory system, the maxima of one simulation trace may eventually coincide with the minima of another, such that their average is a constant non-oscillatory value.

To characterise the behaviour of our abstractions we therefore adopt the frequency domain technique proposed in [23] and used in [17]. In particular, we apply the discrete Fourier transform (DFT) to simulation traces, in order to transform them from the time domain to the frequency domain. The resulting individual frequency spectra, comprising ordered sets of frequency components, may be combined into a single average spectrum. Once the behaviour has been characterised in this way, it is possible to compare it with the average spectra of other systems or abstractions.

The Fourier transform is linear and reversible, hence the resulting complex frequency spectra (i.e., containing both amplitude and phase angle components) are an adequate dual of what is seen in the time domain. Amplitude spectra (without considering phase) are common in the physics and engineering literature because the effects of phase are somewhat non-intuitive and phase is not in general independent of amplitude (i.e., the phase is partially encoded in the amplitude). We have found that the phase component of spectra generated by simulations of CTMCs is uninformative (in the information theoretic sense) and that by excluding the phase component we are able to construct an average frequency spectrum that does not suffer the information loss seen in the time domain. This provides a robust and sensitive empirical characterisation of the average behaviour of a system or of a statistical abstraction.

Our technique can be briefly summarised as follows. Multiple simulation traces are sampled at a suitable fixed time interval $\delta t$ and converted to complex frequency spectra using an efficient implementation of the DFT:

$$
f_{m}=\sum_{n=0}^{N-1} x_{n} e^{-i \frac{2 \pi m n}{N}}
$$

Here $i$ denotes $\sqrt{-1}, f_{m}$ is the $m^{\text {th }}$ frequency component (of a total of $N$ ) and $x_{n}$ is the $n^{\text {th }}$ time sample (of $N$ ) of a given system variable. By virtue of consistent sampling times, the $N$ frequencies in each complex spectrum are the same and may be combined to give a mean distribution. Since the DFT is a linear transformation, the mean of the complex spectra is equivalent to the DFT of the mean of the time series. Hence, to avoid the information loss seen in the time domain, we calculate the mean of the amplitudes of the complex spectra, thus excluding phase.

The values of $N$ and $\delta t$ must be chosen such that the resulting average spectrum encapsulates all the high and low frequencies seen in the interesting behaviour. In practice, the values are either pre-defined or learned from the behaviour seen in a few initial simulations, according to the following considerations. 
$N \delta t$ is the overall time that the system is observed and must obviously be sufficiently long to see all behaviour of interest. Equivalently, $(N \delta t)^{-1}$ is the minimum frequency resolution (the difference between frequencies in the spectrum) and must be small enough to adequately capture the lowest interesting frequency in the behaviour. To make low frequency spectral components appear more distinct (and be more significant in the average spectrum), it may be useful to make $N \delta t$ much longer than the minimum time to see all behaviour (e.g., double), however this must be balanced against the cost of simulation and the cost of calculating (3) (typically $O(N \log N)$ ).

The quantity $(2 \delta t)^{-1}$ defines the maximum observable frequency (the Nyquist frequency) and must be chosen to capture the highest frequency of interest in the behaviour. The theoretical spectrum of an instant discrete transition has frequency components that extend to infinity, however the amplitude of the spectrum decreases with increasing frequency. In a stochastic context, the highest frequency components are effectively hidden below the noise floor created by the stochasticity. The practical consequence is that $\delta t$ may be made much greater than the minimum transition time in the simulation, without losing information about the average behaviour.

In summary, to encapsulate the broadest range of frequencies in the average spectrum it is generally desirable to decrease $\delta t$ and increase $N \delta t$. However, setting the value of $\delta t$ too low may include a lot of uninteresting noise in the spectrum, while setting $N \delta t$ too large may include too much uninteresting low frequency behaviour. In both cases there is increased computational cost.

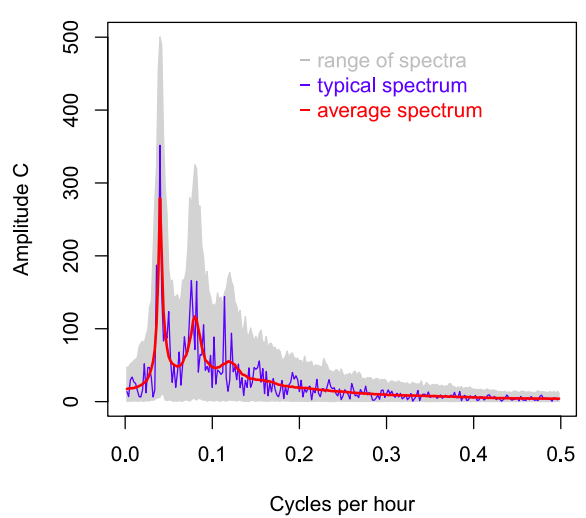

Figure 1. Frequency spectra of protein complex (C) in genetic oscillator [24].

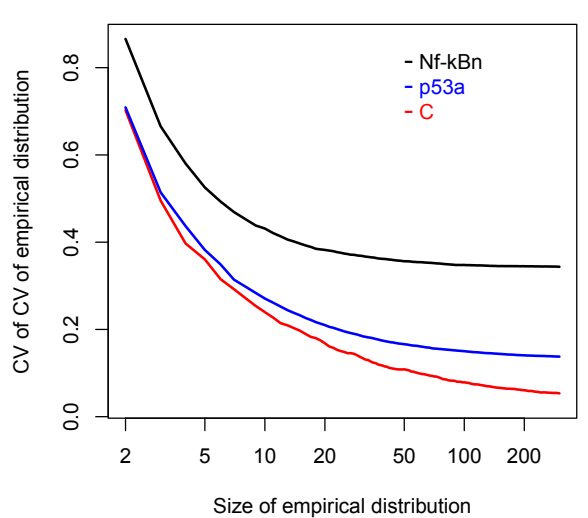

Figure 2. Convergence of empirical distribution abstractions.

To quantify similarity of behaviour, in this paper we use the discrete space version of the Kolmogorov-Smirnov (K-S) statistic [21] to measure the distance between average amplitude spectra. Intuitively, the K-S statistic is the maximum 
absolute difference between two cumulative distributions. This gives a value in the interval $[0,1]$, where 0 corresponds to identical distributions. Heuristically, we consider two distributions to be "close" when the K-S statistic is less than 0.1. Fig. 1 illustrates our technique of frequency domain analysis applied to 1000 simulations of the protein complex (denoted C) in the genetic oscillator of [24].

\subsection{Adequacy of Empirical Abstractions}

In Fig. 1 we see that spectrum of an individual simulation trace is noisy, but the mean is apparently smooth. In fact, the average frequency spectrum comprises discrete points, but because of random phase shifts between simulation traces of CTMCs, there is a strong correlation between adjacent frequencies. To formalise this, using the notation of (3), we denote a frequency magnitude spectrum as $\bigcup_{m=0}^{N-1}\left|f_{m}\right|$, where $\left|f_{m}\right|$ is the magnitude of frequency component $m$. The mean spectrum of an empirical abstraction containing $M$ simulation traces is thus written $\bigcup_{m=0}^{N-1} \frac{1}{M} \sum_{j=1}^{M}\left|f_{m}^{(j)}\right|$, where $\left|f_{m}^{(j)}\right|$ is the magnitude of frequency component $m$ in spectrum $j$. Using the notion of coefficient of variation (CV, defined as the standard deviation divided by the mean), to quantify the a priori adequacy of an empirical distribution abstraction we define the metric

$$
\operatorname{CV}\left(\bigcup_{m=0}^{N-1} \operatorname{CV}\left(\bigcup_{j=1}^{M}\left|f_{m}^{(j)}\right|\right)\right)
$$

The CV is a normalised measure of dispersion (variation). $\mathrm{CV}\left(\bigcup_{j=1}^{M}\left|f_{m}\right|\right)$ is then the normalised dispersion of the data that generates average spectral point $m$. Normalisation is necessary to give equal weight to spectral points having high and low values, which have equal significance in this context. Equation (4) is the normalised dispersion of the normalised dispersions of all the spectral points. The outer normalisation aims to make the metric neutral with respect to subsystems having different absolute levels of stochasticity.

Figure 2 illustrates (4) applied to the empirical abstractions of our case study (NF-kBn and p53a), as well as to the data that generated Fig. 1. Noting the logarithmic x-scale, we observe that the curves have an apprent "corner", such that additional simulations eventually make little difference. The figure suggests that 100 simulation traces will be sufficient for the empirical abstractions of our case study. An empirical abstraction of protein complex $\mathrm{C}$ appears to require at least 200 simulation traces.

\subsection{Critical Abstractions}

Our metric allows us to construct empirical abstractions that encapsulate a notion of the typical behaviour of an external system, in an incremental way. However, certain properties of the core system may be critically dependent on behaviour that is atypical in a "general purpose" abstraction. To identify when 
it is necessary to improve an empirical abstraction, we provide the following indicator.

We consider the empirical abstraction of an arbitrary external subsystem and assume that it contains $N_{A}$ independently generated simulation traces. After performing SMC with $N$ simulations of the complete system, we observe that $n$ satisfy the property, giving $n / N$ as the estimate of the probability that the system satisfies the property. Each simulation requires a sample trace to be drawn from the empirical distribution, hence $n$ samples from the empirical abstraction were "successful". These $n$ samples are not necessarily different (they cannot be if $n>N_{A}$ ), so by $n_{A}$ we denote the number of different samples that were successful. Assuming $N$ to be sufficiently large, we can say that if $n_{A} / N_{A}>n / N$ the external subsystem is relatively unimportant and the abstraction is adequate. Intuitively, the core "restricts" the probability of the external subsystem. If $n_{A} / N_{A} \leq n / N$, however, the property may be critically dependent on the external subsystem, because a smaller proportion of the behaviour of the subsystem satisfies the property than the system as a whole. This condition indicates that the abstraction may not be adequate for the particular property. Moreover, if the absolute value of $n_{A}$ is low, the statistical confidence of the overall estimate is reduced. To improve the abstraction, as well as the overall efficiency with respect to the property, we borrow techniques from importance splitting [18].

The general idea is to increase the occurrence of traces in the abstraction that satisfy the property, then compensate the estimate by the amount their occurrence was increased. If the property of the subsystem that allows the core system to satisfy the property is known (call it the 'subproperty'), we may construct an efficient empirical abstraction that guarantees $n_{A} / N_{A} \geq n / N$ in the following way. SMC is performed on the subsystem using the subproperty, such that only traces that satisfy the subproperty are used in the abstraction. Any properties of the core using this abstraction are conditional on the subproperty and any estimates must be multiplied by the estimated probability of the subproperty with respect to the subsystem (call this the 'subprobability'). In the case of multiple subsystems with this type of abstraction, the final estimate must be multiplied by the product of all subprobabilities.

Using these ideas it is possible to create a set of high performance abstractions optimised to verify rare critical behaviour of complex evolving systems.

\section{Case Study}

Biological systems are an important and challenging area of interest for formal verification (e.g., [15]). The challenges arise from complexity, scale and the lack of complete information. In contrast to the verification of man-made systems, where it is usual to check behaviour with respect to an intended specification, formal verification of biological systems is often used to find out how they work or to hypothesise unknown interactions. Hence, it is not the actual system that 
evolves, but the model of the system, and the task is to ensure that modifications do not affect the critical function of existing parts.

To demonstrate our techniques we consider a biological model of coupled oscillatory systems [17]. The model pre-dates the present work and was constructed to hypothesise elemental reactions linking important biological subsystems, based on available experimental evidence. A core model of the cell cycle receives external oscillatory signals from models of protein families NF- $\kappa \mathrm{B}$ and p53. Although the semantics of the model is chemical reactions, it is nevertheless typical of many computational systems. The model contains 93 commands and a crude estimate of the total number of states is $10^{174}$ (estimated from the range of values seen in simulations). An overview of the model is given in Appendix A, with precise technical details given in Tables 1 to 5 . A more detailed biological description is given in [17].

\subsection{Results}

The external subsystems in our biological case study are themselves simplifications of very much larger systems, but we are nevertheless able to make substantial improvements in performance by abstraction. The improvements would be greater still if we were to consider the unsimplified versions of the external subsystems. The following numerical results are the average of hundreds of simulations and may be assumed to be within $\pm 5 \%$ of the extreme values.

The behavioural phenomena in the case study require simulations of approximately 67 hours of simulated time, corresponding to approximately $72 \times 10^{6}$ simulation steps in the complete model. Of this, approximately $40 \times 10^{6}$ steps are due to the p53 system and approximately $20 \times 10^{6}$ steps are due to the NF- $\kappa \mathrm{B}$ system. The abstracted traces of p53a are approximately $5.2 \times 10^{6}$ steps, while the abstracted traces of NF-kBn are approximately $1.3 \times 10^{6}$ steps. Hence, an equivalent simulation using these abstractions with Algorithm 1 requires only about $18.5 \times 10^{6}$ steps. Moreover, because we remove 41 commands from the model, each step takes approximately 52/93 as much time. Overall, we make a worthwhile seven-fold improvement in simulation performance.

On the basis of the results presented in Fig. 2, we suppose that 100 traces are adequate for our empirical distributions. This number is likely to be more than an order of magnitude fewer than the number of simulations required for SMC, so there is a saving in the cost of checking a single property, even when the cost of creating the abstractions is included. Subsequent savings are greater. By considering only the output variables p53a and NF-kBn, the size of each trace is reduced by factors of approximately 27 and 85, respectively. Without compression, the empirical abstractions for p53a and NF-kBn occupy approximately 2.4 and 0.6 gigabytes of memory, respectively. This is tractable with current hardware, however we anticipate that a practical implementation will compress the empirical abstractions using an algorithm optimised for fast decompression, e.g., using the Lempel-Ziv-Oberhumer (LZO) library. ${ }^{1}$

\footnotetext{
${ }^{1}$ www.oberhumer.com/opensource/lzo
} 


\subsection{Gaussian Process Abstraction}

In this section we report promising results using a memory-efficient form of statistical abstraction based on Gaussian processes. If we can generate traces that are statistically indistinguishable from samples of the original distribution, we can avoid the storage costs of an empirical distribution. Gaussian processes are popular in machine learning [22] and work by constructing functions that model the time evolution of the mean and covariance of multivariate Gaussian random variables.

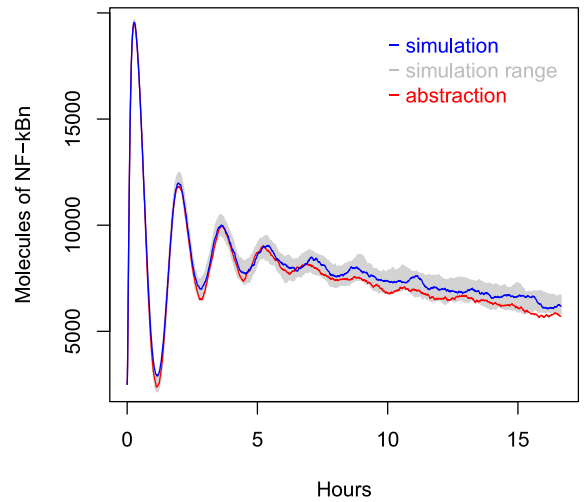

Figure 3. Traces of NF-kBn generated by simulation and abstraction.

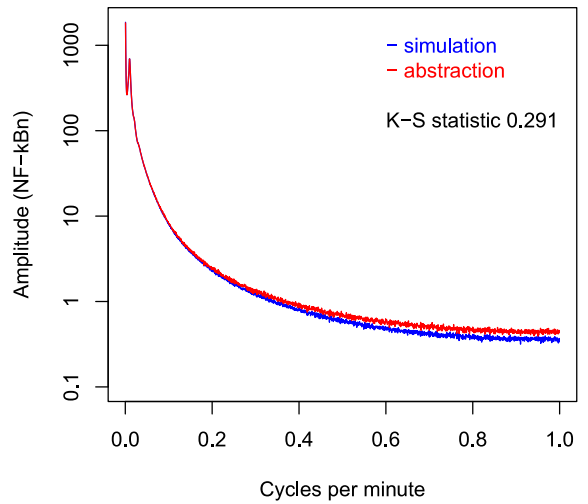

Figure 4. Average spectra of simulation and abstraction of NF-kBn.

Since we wish to judge our abstraction with frequency domain analysis, we construct a simple process that generates sampled traces directly. This is sufficient to reveal the potential and shortfalls of the approach. We assume a training set of $M$ sampled simulation traces of the output variable of a subsystem, denoted $\bigcup_{j=1}^{M}\left(x_{n}^{(j)}\right)_{n=0}^{N-1}$, where $x_{n}^{(j)}$ is the $n^{\text {th }}$ sample of variable $x$ in simulation trace $j$. From this we construct a sequence of Gaussian random variables

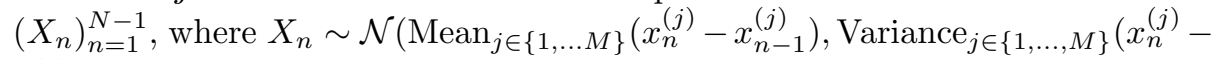
$\left.x_{n-1}^{(j)}\right)$ ). Each $X_{n}$ thus models the change in values from sample $n-1$ to sample $n$. A trace $(y)_{n=0}^{N-1}$ may be generated from this abstraction by setting $y_{0}=x_{0}$ (the initial value) and iteratively calculating $y_{n}=y_{n-1}+\xi_{n}$, where $\xi_{n}$ is a sample from $X_{n}$.

To judge the performance of our abstraction we measure the K-S distance between empirical distributions generated by the original system and by the Gaussian processes. We then compare this with the K-S distance between two empirical distributions generated by the original system (the 'self distance'). In this investigation all distributions contain 100 traces. With infinitely large distributions the expected self distance is zero, but the random variation between finite 
distributions causes the value to be higher. With 100 traces the self distances for p53a and NF-kBn are typically $0.02 \pm 0.01$. The K-S distances between the Gaussian process abstractions and empirical distributions of simulations form the original systems are typically 0.03 for $\mathrm{p} 53 \mathrm{a}$ and 0.3 for NF-kBn. Using this metric, the p53a abstraction is almost indistinguishable from the original, but the abstraction for NF-kBn is not adequate. Figs. 3 and 4 illustrate the performance of the Gaussian process abstraction for NF-kBn. At this scale the p53a abstraction is visually indistinguishable from the original and is therefore not shown.

\section{Challenges and Prospects}

Empirical distribution abstractions are simple to construct, provide sample traces that are correct with respect to the causality of the systems they abstract, but are memory-intensive. In contrast, Gaussian processes offer a memory-efficient away to abstract external systems, but do not implicitly guarantee correct causality and their parameters must be learned from no fewer simulations than would be required for an empirical distribution. Despite this, Gaussian processes seem to offer the greatest potential for development, as a result of their scalability.

Our preliminary results suggest that it may be possible to create very good abstractions with Gaussian processes, but that our current simplistic approach will not in general be adequate. Our ongoing work will therefore investigate more sophisticated processes, together with ways to guarantee that their behaviour respects the causality of the systems they abstract. Their increased complexity will necessarily entail more sophisticated learning techniques, whose computational cost must also be included when considering efficiency.

A substantial future challenge is to adapt our techniques to systems with bidirectional communication between components. In this context empirical distributions are unlikely to be adequate, since the abstractions would be required to change in response to input signals. One plausible approach is to construct Gaussian processes parametrised by functions of input signals.

\section{References}

1. C. Baier, B. Haverkort, H. Hermanns, and J. Katoen. Model-checking algorithms for continuous-time markov chains. Software Engineering, IEEE Transactions on, 29(6):524-541, 2003.

2. A. Basu, S. Bensalem, M. Bozga, B. Caillaud, B. Delahaye, and A. Legay. Statistical abstraction and model-checking of large heterogeneous systems. In Formal Techniques for Distributed Systems, pages 32-46. Springer, 2010.

3. A. Biere, A. Cimatti, E. Clarke, and Y. Zhu. Symbolic model checking without BDDs. In W. Cleaveland, editor, Tools and Algorithms for the Construction and Analysis of Systems, volume 1579 of LNCS, pages 193-207. Springer, 1999.

4. L. Bortolussi and G. Sanguinetti. Learning and designing stochastic processes from logical constraints. In K. Joshi, M. Siegle, M. Stoelinga, and P. R. D'Argenio, editors, Quantitative Evaluation of Systems, volume 8054 of LNCS, pages 89-105. Springer, 2013. 
5. J. R. Burch, E. M. Clarke, K. L. McMillan, D. L. Dill, and L.-J. Hwang. Symbolic model checking: $10^{20}$ states and beyond. Information and computation, 98(2):142$170,1992$.

6. E. M. Clarke, E. A. Emerson, and J. Sifakis. Model checking: algorithmic verification and debugging. Commun. ACM, 52(11):74-84, Nov. 2009.

7. E. M. Clarke, O. Grumberg, and D. E. Long. Model checking and abstraction. $A C M$ Transactions on Programming Languages and Systems (TOPLAS), 16(5):15121542, 1994.

8. P. Cousot and R. Cousot. Abstract interpretation: a unified lattice model for static analysis of programs by construction or approximation of fixpoints. In Proceedings of the 4th ACM SIGACT-SIGPLAN symposium on Principles of programming languages, pages 238-252. ACM, 1977.

9. E. W. Dijkstra. Guarded commands, nondeterminacy and formal derivation of programs. Commun. ACM, 18:453-457, August 1975.

10. M. Gibson and J. Bruck. Efficient exact stochastic simulation of chemical systems with many species and many channels. J. of Physical Chemistry A, 104:1876, 2000.

11. D. Gillespie. Stochastic simulation of chemical kinetics. Annual Review of Physical Chemistry, 58(1):35-55, 2007.

12. D. T. Gillespie. A general method for numerically simulating the stochastic time evolution of coupled chemical reactions. Journal of Computational Physics, 22(4):403 - 434, 1976.

13. D. T. Gillespie. A rigorous derivation of the chemical master equation. Physica A, 188:404-425, 1992.

14. P. Godefroid. Using partial orders to improve automatic verification methods. In Computer-Aided Verification, pages 176-185. Springer, 1991.

15. J. Heath and et al. Probabilistic model checking of complex biological pathways. Theoretical Computer Science, 391:239-257, 2008.

16. M. Hinchey and L. Coyle. Evolving critical systems. In Engineering of Computer Based Systems (ECBS), 2010 17th IEEE International Conference and Workshops on, pages 4-4, March 2010.

17. A. Ihekwaba and S. Sedwards. Communicating oscillatory networks: frequency domain analysis. BMC Systems Biology, 5(1):203, 2011.

18. C. Jegourel, A. Legay, and S. Sedwards. Importance splitting for statistical model checking rare properties. In N. Sharygina and H. Veith, editors, Computer Aided Verification, volume 8044 of LNCS, pages 576-591. Springer, 2013.

19. J. G. Kemeny, A. W. Knapp, and J. L. Snell. Denumerable markov chains. Springer, 1976.

20. M. Okamoto. Some inequalities relating to the partial sum of binomial probabilities. Annals of the Institute of Statistical Mathematics, 10:29-35, 1959.

21. A. N. Pettitt and M. A. Stephens. The Kolmogorov-Smirnov Goodness-of-Fit Statistic with Discrete and Grouped Data. Technometrics, 19(2):205-210, 1977.

22. C. E. Rasmussen and C. K. I. Williams. Gaussian Processes for Machine Learning. The MIT Press, 2006.

23. S. Sedwards. A Natural Computation Approach To Biology: Modelling Cellular Processes and Populations of Cells With Stochastic Models of P Systems. PhD thesis, University of Trento, 2009.

24. J. M. G. Vilar, H. Y. Kueh, N. Barkai, and S. Leibler. Mechanisms of noiseresistance in genetic oscillators. Proceedings of the National Academy of Sciences, 99(9):5988-5992, 2002.

25. A. Wald. Sequential Tests of Statistical Hypotheses. Annals of Mathematical Statistics, 16(2):117-186, 1945. 


\section{A Model of Coupled Oscillatory Systems}

Fig. 5 illustrates the direct effect that one chemical species in the model has on another. Each node represents a state variable, whose value records the instantaneous number of molecules of a chemical species. The edges in the graph are directional, having a source and destination node. Influence that acts in both directions is represented by a bi-directional edge. Positive influence implies that increasing the number of source molecules will increase the number of destination molecules. The presence of an edge in the diagram indicates the existence of a command in which the source species variable appears in the rate and the destination species variable appears in the action. The variables that we use to abstract the external systems, namely p53a and NF-kBn, are highlighted in red.

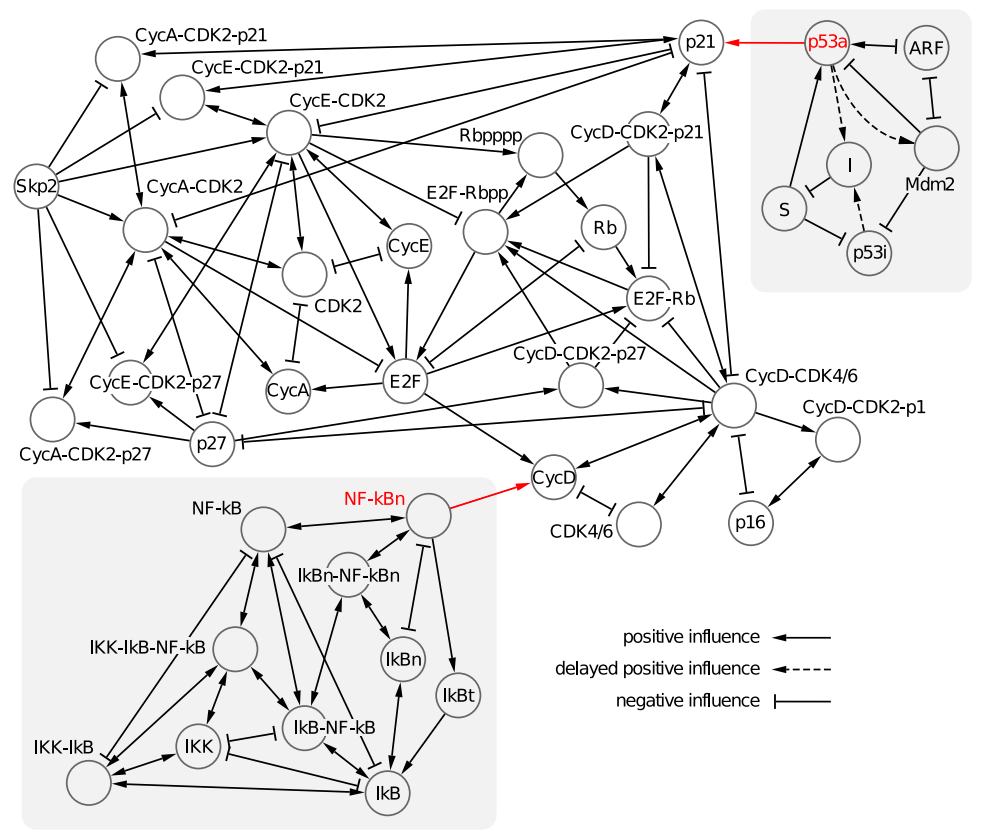

Figure 5. Direct influence of variables in the case study. External systems are shown on grey backgrounds. Variables in red are used in abstractions.

The precise technical details of the model are given in Tables 1 to 5 . Table 2 gives the reaction scheme of the core system (the cell cycle). Tables 1 and 3 contain the reactions schemes of the external systems (NF- $\kappa \mathrm{B}$ and $\mathrm{p} 53$, respectively). Table 4 gives the initial numbers of molecules of each species and Table 5 gives the values of the constants used.

The model is given in terms of chemical reactions for compactness and to be compatible with previous work on these systems. Reactions have the form 
reactants $\rightarrow$ products, where reactants and products are possibly empty multisets of chemical species, described by the syntax $\emptyset \mid \mathrm{S}\{$ "+" S \}, in which $\emptyset$ denotes the empty multiset and $\mathrm{S}$ is the name of a chemical species. The semantics of reactions assumes that the system contains a multiset of molecules. A reaction is enabled if reactants is a subset of this multiset. An enabled reaction is executed by removing reactants from the system and simultaneously adding products.

The rate at which a reaction is executed is stochastic. The majority of the reactions in our model are 'elemental' [11], working by 'mass action kinetics' according to fundamental physical laws [13]. Hence, under the assumption that the system is 'well stirred' [13], the rate at which a reaction is executed is given by the product of some rate constant $k$ and the number of ways that reactants may be removed from the system. Elemental reactions in our model are thus converted to commands according to the following table.

\begin{tabular}{|c|c|}
\hline Reaction pattern & Command (guard, rate, action $)$ \\
\hline \hline$\emptyset \rightarrow \mathrm{A}$ & $($ true $, k, A=A+1)$ \\
\hline $\mathrm{A} \rightarrow \mathrm{B}$ & $(A>0, k A, A=A, A=A-1 ; B=B+1)$ \\
\hline $\mathrm{A} \rightarrow \mathrm{B}+\mathrm{C}$ & $(A>0 \wedge B>0, k A B, A=A-1 ; B=B-1 ; C=C+1)$ \\
\hline $\mathrm{A}+\mathrm{B} \rightarrow \mathrm{C}$ & $(A>0 \wedge B>0, k A B, A=A-1 ; B=B-1 ; C=C+1 ; D=D+1)$ \\
\hline $\mathrm{A}+\mathrm{B} \rightarrow \mathrm{C}+\mathrm{D}$ & $(A>C+1)$ \\
\hline
\end{tabular}

A few of the reactions are abstractions of more complex mechanisms, using Michaelis-Menten dynamics, Hill coefficients or delays. The semantics of their execution is the same, so the guard and action given above are correct, but the rate is given by an explicit function. Delays appear in the rate as a function of a molecular species $\mathrm{S}$ and a delay time $\tau$. The value of this function at time $t$ is the number of molecules of $\mathrm{S}$ at time $t-\tau$.

The reaction rate constants and initial values have been inferred using ODE models that consider concentrations, rather than numbers of molecules. To convert the initial concentrations to molecules, they must be multiplied by a discretisation constant, alpha, having the dimensions of volume. Specifically, alpha is the product of Avogadro's number and the volume of a mammalian cell. The rate constants must also be transformed to work with numbers of molecules. The rate constants of reactions of the form $\emptyset \rightarrow \cdots$ must be multiplied by alpha. The rate constants of reactions of the form $\mathrm{A}+\mathrm{B} \rightarrow \cdots$ must be divided by alpha. The rate constants of reactions of the form $\mathrm{A} \rightarrow \cdots$ may be used unaltered. In the case of explicit functions that are the product of a rate constant and a number of molecules raised to some power $\mathrm{h}$, the value of the constant must be divided by alpha ${ }^{\mathrm{h}-1}$. 


\begin{tabular}{|c|c|}
\hline Reaction & Rate constant or [function] \\
\hline $\mathrm{IkB} \rightarrow \emptyset$ & $\operatorname{kdeg} 1$ \\
\hline $\mathrm{IkB} \rightarrow \mathrm{IkBn}$ & ktp1 \\
\hline $\mathrm{IkB}+\mathrm{NFkB} \rightarrow \mathrm{IkBNFkB}$ & la4 \\
\hline $\mathrm{IkBt} \rightarrow \mathrm{IkB}+\mathrm{IkBt}$ & ktr1 \\
\hline $\mathrm{IkBn} \rightarrow \mathrm{IkB}$ & ktp2 \\
\hline $\mathrm{IkBn}+\mathrm{NFkBn} \rightarrow \mathrm{nIkBNFkB}$ & la4 \\
\hline $\mathrm{IkBn} \rightarrow \emptyset$ & kdeg1 \\
\hline $\mathrm{nIkBNFkB} \rightarrow \mathrm{IkBn}+\mathrm{NFkBn}$ & kd4 \\
\hline nIkBNFkB $\rightarrow$ IkBNFkB & $\mathrm{k} 2$ \\
\hline nIkBNFkB $\rightarrow$ NFkBn & kdeg5 \\
\hline $\mathrm{IkBNFkB} \rightarrow \mathrm{nIkBNFkB}$ & k3 \\
\hline $\mathrm{IkBt} \rightarrow \emptyset$ & ktr3 \\
\hline$\emptyset \rightarrow \mathrm{IkBt}$ & $\operatorname{tr} 2 \mathrm{a}$ \\
\hline$\emptyset \rightarrow \mathrm{IkBt}$ & {$\left[\operatorname{tr} 2 \times(\mathrm{NFkBn})^{\mathrm{h}}\right]$} \\
\hline $\mathrm{IkBNFkB} \rightarrow \mathrm{IkB}+\mathrm{NFkB}$ & $\mathrm{kd} 4$ \\
\hline $\mathrm{IkBNFkB} \rightarrow \mathrm{NFkB}$ & $\operatorname{kdeg} 4$ \\
\hline $\mathrm{IKK}+\mathrm{IkB} \rightarrow \mathrm{IKKIkB}$ & la1 \\
\hline $\mathrm{IKK}+\mathrm{IkBNFkB} \rightarrow \mathrm{KIkBNFkB}$ & la7 \\
\hline $\mathrm{IKK} \rightarrow \emptyset$ & k02 \\
\hline $\mathrm{IKKIkB} \rightarrow \mathrm{IKK}+\mathrm{IkB}$ & kd1 \\
\hline IKKIkB $\rightarrow$ IKK & kr1 \\
\hline $\mathrm{IKKIkB}+\mathrm{NFkB} \rightarrow \mathrm{KIkBNFkB}$ & la4 \\
\hline $\mathrm{KIkBNFkB} \rightarrow \mathrm{IKK}+\mathrm{IkBNFkB}$ & $\mathrm{kd} 2$ \\
\hline $\mathrm{KIkBNFkB} \rightarrow \mathrm{IKKIkB}+\mathrm{NFkB}$ & $\mathrm{kd} 4$ \\
\hline KIkBNFkB $\rightarrow$ NFkB + IKK & kr4 \\
\hline $\mathrm{NFkB} \rightarrow \mathrm{NFkBn}$ & $\mathrm{k} 1$ \\
\hline $\mathrm{NFkBn} \rightarrow \mathrm{NFkB}$ & k01 \\
\hline
\end{tabular}

Table 1. NF- $\kappa \mathrm{B}$ reactions. 


\begin{tabular}{|c|c|}
\hline Reaction & Rate constant or [function] \\
\hline CycDCDK $46 \rightarrow$ CDK 46 & $\mathrm{R} 1$ \\
\hline CycDCDK46 + p16 $\rightarrow$ CycDCDp16 & $\mathrm{R} 29$ \\
\hline $\mathrm{CycDCDK} 46+\mathrm{p} 27 \rightarrow \mathrm{CycDCDp} 27$ & $\mathrm{R} 6$ \\
\hline $\mathrm{CycDCDK} 46 \rightarrow \mathrm{CDK} 46+\mathrm{CycD}$ & $\mathrm{R} 21 \mathrm{~b}$ \\
\hline $\mathrm{CycD}+\mathrm{CDK} 46 \rightarrow \mathrm{CycDCDK} 46$ & $\mathrm{R} 21 \mathrm{a}$ \\
\hline $\mathrm{CDK} 46 \rightarrow \emptyset$ & R32 \\
\hline $\mathrm{CycACDK} 2+\mathrm{E} 2 \mathrm{~F} \rightarrow \mathrm{CycACDK} 2$ & $\mathrm{R} 15$ \\
\hline$\emptyset \rightarrow \mathrm{E} 2 \mathrm{~F}$ & $\mathrm{R} 43$ \\
\hline $\mathrm{E} 2 \mathrm{~F} \rightarrow \mathrm{E} 2 \mathrm{~F}+\mathrm{E} 2 \mathrm{~F}$ & $\mathrm{R} 42$ \\
\hline $\mathrm{CycE} \rightarrow \emptyset$ & $\mathrm{R} 26$ \\
\hline $\mathrm{E} 2 \mathrm{~F} \rightarrow \mathrm{CycE}+\mathrm{E} 2 \mathrm{~F}$ & $\mathrm{R} 2$ \\
\hline $\mathrm{CycECDK} 2 \rightarrow \mathrm{CDK} 2$ & R3 \\
\hline $\mathrm{CycECDK} 2 \rightarrow \mathrm{CDK} 2+\mathrm{CycE}$ & $\mathrm{R} 24 \mathrm{~b}$ \\
\hline $\mathrm{CDK} 2+\mathrm{CycE} \rightarrow \mathrm{CycECDK} 2$ & $\mathrm{R} 24 \mathrm{a}$ \\
\hline $\mathrm{CDK} 2+\mathrm{CycA} \rightarrow \mathrm{CycACDK} 2$ & $\mathrm{R} 25 \mathrm{a}$ \\
\hline $\mathrm{CDK} 2 \rightarrow \emptyset$ & R33 \\
\hline $\mathrm{CycA} \rightarrow \emptyset$ & $\mathrm{R} 27$ \\
\hline $\mathrm{E} 2 \mathrm{~F} \rightarrow \mathrm{CycA}+\mathrm{E} 2 \mathrm{~F}$ & $\mathrm{R} 4$ \\
\hline $\mathrm{CycACDK} 2 \rightarrow \mathrm{CDK} 2$ & R5 \\
\hline $\mathrm{CycACDK} 2 \rightarrow \mathrm{CycA}+\mathrm{CDK} 2$ & $\mathrm{R} 25 \mathrm{~b}$ \\
\hline $\mathrm{p} 27+\mathrm{CycECDK} 2 \rightarrow \mathrm{CycECDp} 27$ & $\mathrm{R} 7$ \\
\hline $\mathrm{p} 27+\mathrm{CycACDK} 2 \rightarrow \mathrm{CycACDp} 27$ & $\mathrm{R} 8$ \\
\hline$\emptyset \rightarrow \mathrm{p} 27$ & $\mathrm{R} 20$ \\
\hline CycECDp27 + Skp2 $\rightarrow$ Skp2 + CycECDK2 & $\mathrm{R} 9$ \\
\hline CycACDp27 + Skp2 $\rightarrow$ Skp2 + CycACDK2 & $\mathrm{R} 10$ \\
\hline Skp2 $\rightarrow \emptyset$ & $\mathrm{R} 34$ \\
\hline$\emptyset \rightarrow \mathrm{Skp} 2$ & R31 \\
\hline $\mathrm{Rb} \rightarrow \emptyset$ & $\mathrm{R} 18$ \\
\hline $\mathrm{Rb}+\mathrm{E} 2 \mathrm{~F} \rightarrow \mathrm{E} 2 \mathrm{FRb}$ & $\mathrm{R} 11$ \\
\hline$\emptyset \rightarrow \mathrm{Rb}$ & $\mathrm{R} 17$ \\
\hline $\mathrm{Rbpppp} \rightarrow \mathrm{Rb}$ & $\mathrm{R} 16$ \\
\hline $\mathrm{CycDCDK} 46+\mathrm{E} 2 \mathrm{FRb} \rightarrow \mathrm{E} 2 \mathrm{FRbpp}+\mathrm{CycDCDK} 46$ & $\mathrm{R} 12$ \\
\hline CycDCDp27 + E2FRb $\rightarrow$ E2FRbpp + CycDCDp27 & $\mathrm{R} 13$ \\
\hline CycDCDp21 + E2FRb $\rightarrow$ E2FRbpp + CycDCDp21 & $\mathrm{R} 41$ \\
\hline $\mathrm{E} 2 \mathrm{FRbpp}+\mathrm{CycECDK} 2 \rightarrow \mathrm{CycECDK} 2+\mathrm{Rbpppp}+\mathrm{E} 2 \mathrm{~F}$ & $\mathrm{R} 14$ \\
\hline CycDCDp16 $\rightarrow$ p16 & $\mathrm{R} 19$ \\
\hline $\mathrm{p} 16 \rightarrow \emptyset$ & $\mathrm{R} 23$ \\
\hline$\emptyset \rightarrow \mathrm{p} 16$ & $\mathrm{R} 28$ \\
\hline $\mathrm{CycD} \rightarrow \emptyset$ & $\mathrm{R} 22$ \\
\hline $\mathrm{E} 2 \mathrm{~F} \rightarrow \mathrm{CycD}+\mathrm{E} 2 \mathrm{~F}$ & $\mathrm{R} 44$ \\
\hline$\emptyset \rightarrow \mathrm{CycD}$ & R30a \\
\hline $\mathrm{p} 21+\mathrm{CycDCDK} 46 \rightarrow \mathrm{CycDCDp} 21$ & $\mathrm{R} 35 \mathrm{a}$ \\
\hline $\mathrm{p} 21+\mathrm{CycECDK} 2 \rightarrow \mathrm{CycECDp} 21$ & $\mathrm{R} 36 \mathrm{a}$ \\
\hline $\mathrm{p} 21+\mathrm{CycACDK} 2 \rightarrow \mathrm{CycACDp} 21$ & $\mathrm{R} 37 \mathrm{a}$ \\
\hline$\emptyset \rightarrow \mathrm{p} 21$ & $\mathrm{R} 40 \mathrm{a}$ \\
\hline $\mathrm{CycDCDp} 21 \rightarrow \mathrm{p} 21+\mathrm{CycDCDK} 46$ & $\mathrm{R} 35 \mathrm{~b}$ \\
\hline CycECDp21 $\rightarrow$ p21 + CycECDK 2 & $\mathrm{R} 36 \mathrm{~b}$ \\
\hline Skp2 + CycECDp21 $\rightarrow$ CycECDK2 + Skp2 & R38 \\
\hline CycACDp21 $\rightarrow$ p21 + CycACDK2 & $\mathrm{R} 37 \mathrm{~b}$ \\
\hline Skp2 + CyCACDp21 $\rightarrow$ CycACDK2 + Skp2 & R39 \\
\hline$\emptyset \rightarrow \mathrm{CycD}$ & {$\left[\mathrm{R} 30 \mathrm{~b} \times(\mathrm{NFkBn})^{\mathrm{h}}\right]$} \\
\hline $\mathrm{p} 53 \mathrm{a} \rightarrow \mathrm{p} 21+\mathrm{p} 53 \mathrm{a}$ & $\mathrm{R} 40 \mathrm{~b}$ \\
\hline
\end{tabular}

Table 2. Cell cycle reactions. 


\begin{tabular}{ll} 
Reaction & Rate constant or [function] \\
\hline $\mathrm{p} 53 \mathrm{i}+\mathrm{Mdm} 2 \rightarrow \mathrm{Mdm} 2$ & kap53i \\
$\emptyset \rightarrow \mathrm{p} 53 \mathrm{i}$ & $\mathrm{kbp} 53 \mathrm{i}$ \\
$\mathrm{p} 53 \mathrm{a}+\mathrm{Mdm} 2 \rightarrow \mathrm{Mdm} 2$ & $\mathrm{kap} 53 \mathrm{a}$ \\
$\mathrm{p} 53 \mathrm{i} \rightarrow \mathrm{p} 53 \mathrm{a}$ & {$[\mathrm{w} \times(\mathrm{Sn} /(\mathrm{Sn}+\mathrm{Ts})) \times \mathrm{p} 53 \mathrm{i}]$} \\
$\mathrm{ARF}+\mathrm{p} 53 \mathrm{a} \rightarrow 2 \mathrm{p} 53 \mathrm{a}$ & $\mathrm{R} 46$ \\
$\mathrm{Mdm} 2 \rightarrow \emptyset$ & $\mathrm{kaMdm} 2$ \\
$\mathrm{Mdm} 2+\mathrm{ARF} \rightarrow \emptyset$ & $\mathrm{R} 48$ \\
$\emptyset \rightarrow \mathrm{Mdm} 2$ & {$[\mathrm{kbMdm} 2 \times \operatorname{delay}(\mathrm{p} 53 \mathrm{a}, \mathrm{tau})]$} \\
$\mathrm{I} \rightarrow \emptyset$ & $\mathrm{kai}$ \\
$\emptyset \rightarrow \mathrm{I}$ & {$[\mathrm{kbi} \times($ delay $(\mathrm{p} 53 \mathrm{a}, \mathrm{tau})+$ delay $(\mathrm{p} 53 \mathrm{i}, \mathrm{tau}))]$} \\
$\mathrm{ARF} \rightarrow \emptyset$ & $\mathrm{R} 47$ \\
$\emptyset \rightarrow \mathrm{ARF}$ & $\mathrm{R} 45 \mathrm{a}$ \\
$\mathrm{S}+\mathrm{I} \rightarrow \mathrm{I}$ & $\mathrm{kas}$ \\
$\emptyset \rightarrow \mathrm{S}$ & $\mathrm{kbs} \times \mathrm{e}$ \\
\hline
\end{tabular}

Table 3. p53 reactions.

\begin{tabular}{ll|ll} 
Species & Amount & Species & Amount \\
\hline p53i & 0 & CycECDK2 & 0 \\
p53a & $0.1 \times$ alpha & CDK2 & $2.0 \times$ alpha \\
Mdm2 & $0.15 \times$ alpha & CycA & 0 \\
I & $0.1 \times$ alpha & CycACDK2 & 0 \\
S & 0 & p27 & $1.0 \times$ alpha \\
ARF & 0 & CycDCDp27 & $0.001 \times$ alpha \\
IkB & 0 & CycECDp27 & 0 \\
IkBn & 0 & CycACDp27 & 0 \\
nIkBNFkB & 0 & Skp2 & $1.0 \times$ alpha \\
IkBt & 0 & Rb & $1.0 \times$ alpha \\
IkBNFkB & $0.2 \times$ alpha & E2FRb & $1.95 \times$ alpha \\
IKK & $0.2 \times$ alpha & E2FRbpp & $1.0 \times 10^{-3} \times$ alpha \\
IKKIkB & 0 & Rbpppp & $1.02 \times$ alpha \\
KIkBNFkB & 0 & CycDCDp16 $1.0 \times 10^{-5} \times$ alpha \\
NFkB & 0 & p16 & $1.0 \times$ alpha \\
NFkBn & $0.025 \times$ alpha & CycD & 0 \\
CycDCDK46 & 0 & p21 & 0 \\
CDK46 & $5.0 \times$ alpha & CycDCDp21 0 \\
E2F & 0 & CycECDp21 0 \\
CycE & 0 & CycACDp21 0 \\
\hline \multicolumn{2}{l}{ Table 4. Initial number of molecules. }
\end{tabular}




\begin{tabular}{|c|c|c|c|c|c|}
\hline Name & Value & Name & Value & Name & Value \\
\hline$\overline{\text { alpha }}$ & 100000 & $\mathrm{R} 8$ & $7.0 \times 10^{-2} /$ alpha & R35b & $5.0 \times 10^{-3}$ \\
\hline $\mathrm{h}$ & 2 & R9 & $0.225 /$ alpha & R36a & $1.0 \times 10^{-2} / \mathrm{alpha}$ \\
\hline kdeg1 & 0.16 & R10 & $2.5 \times 10^{-3} /$ alpha & R36b & $1.75 \times 10^{-4}$ \\
\hline ktp1 & 0.018 & R11 & $5.0 \times 10^{-5} /$ alpha & R37a & $7.0 \times 10^{-2} /$ alpha \\
\hline ktp2 & 0.012 & $\mathrm{R} 12$ & $1.0 \times 10^{-4} /$ alpha & R37b & $1.75 \times 10^{-4}$ \\
\hline ktr1 & 0.2448 & $\mathrm{R} 13$ & $1.0 \times 10^{-2} /$ alpha & R38 & $0.225 /$ alpha \\
\hline $\mathrm{kd} 4$ & 0.00006 & $\mathrm{R} 14$ & 0.073/alpha & R39 & $2.5 \times 10^{-3} /$ alpha \\
\hline la1 & 0.1776/alpha & $\mathrm{R} 15$ & $0.022 /$ alpha & R40a & $5.0 \times 10^{-5} \times$ alpha \\
\hline $\mathrm{kd} 1$ & 0.000888 & R16 & $5.0 \times 10^{-8}$ & $\mathrm{R} 40 \mathrm{~b}$ & $1.0 \times 10^{-3}$ \\
\hline la4 & 30/alpha & $\mathrm{R} 17$ & $5.0 \times 10^{-5} \times$ alpha & R41 & $1.0 \times 10^{-2} /$ alpha \\
\hline $\mathrm{k} 2$ & 0.552 & R19 & $5.0 \times 10^{-2} /$ alpha & $\mathrm{R} 42$ & $1.0 \times 10^{-4}$ \\
\hline k3 & 0.00006 & $\mathrm{R} 20$ & $1.0 \times 10^{-4} \times$ alpha & R43 & $5.0 \times 10^{-5} \times$ alpha \\
\hline $\operatorname{tr} 2 \mathrm{a}$ & $0.000090133 \times$ alpha & $\mathrm{R} 21 \mathrm{a}$ & $2.0 \times 10^{-3} /$ alpha & $\mathrm{R} 44$ & $3.0 \times 10^{-4}$ \\
\hline ktr3 & 0.020733 & $\mathrm{R} 21 \mathrm{~b}$ & $8.0 \times 10^{-3}$ & $\mathrm{R} 45 \mathrm{a}$ & $8.0 \times 10^{-5} \times$ alpha \\
\hline $\operatorname{tr} 2$ & $0.5253 / \mathrm{alpha}^{(\mathrm{h}-1)}$ & $\mathrm{R} 22$ & $7.5 \times 10^{-3}$ & $\mathrm{R} 45 \mathrm{~b}$ & 0.008 \\
\hline $\operatorname{kdeg} 4$ & 0.00006 & $\mathrm{R} 23$ & $5.0 \times 10^{-3}$ & R46 & $2.333 \times 10^{-5} /$ alpha \\
\hline kdeg5 & 0.00006 & $\mathrm{R} 24 \mathrm{a}$ & $8.0 \times 10^{-3} /$ alpha & $\mathrm{R} 47$ & 0.01167 \\
\hline la7 & 6.06/alpha & $\mathrm{R} 24 \mathrm{~b}$ & $3.9 \times 10^{-3}$ & R48 & $1.167 \times 10^{-5} /$ alpha \\
\hline $\mathrm{kd} 2$ & 0.095 & $\mathrm{R} 25 \mathrm{a}$ & $8.0 \times 10^{-3} /$ alpha & kbp53i & $0.015 \times$ alpha \\
\hline kr1 & 0.012 & $\mathrm{R} 25 \mathrm{~b}$ & $4.0 \times 10^{-3}$ & kbMdm2 & 0.01667 \\
\hline $\mathrm{kr} 4$ & 0.22 & $\mathrm{R} 26$ & $2.5 \times 10^{-3}$ & kap53i & 2.333/alpha \\
\hline k1 & 5.4 & $\mathrm{R} 27$ & $5.0 \times 10^{-4}$ & kaMdm2 & 0.01167 \\
\hline k01 & 0.0048 & $\mathrm{R} 28$ & $2.0 \times 10^{-4} \times$ alpha & tau & 80 \\
\hline k02 & 0.0072 & $\mathrm{R} 29$ & $5.0 \times 10^{-4} /$ alpha & kap53a & 0.02333/alpha \\
\hline $\mathrm{R} 1$ & $5.0 \times 10^{-6}$ & R30a & $0.004 \times$ alpha & kas & 0.045/alpha \\
\hline $\mathrm{R} 2$ & $4.5 \times 10^{-3}$ & R30b & $0.9961 /$ alpha $^{(\mathrm{h}-1)}$ & $\mathrm{kbi}$ & 0.01667 \\
\hline $\mathrm{R} 3$ & $5.0 \times 10^{-3}$ & R31 & $5.0 \times 10^{-4} \times$ alpha & kai & 0.01167 \\
\hline $\mathrm{R} 4$ & $2.5 \times 10^{-3}$ & R32 & $8.0 \times 10^{-4}$ & kbs & $0.015 \times$ alpha \\
\hline R5 & $5.0 \times 10^{-4}$ & R33 & $8.0 \times 10^{-4}$ & e & 1 \\
\hline $\mathrm{R} 6$ & $5.0 \times 10^{-4} /$ alpha & R34 & $9.0 \times 10^{-4}$ & $\mathrm{n}$ & 4 \\
\hline $\mathrm{R} 7$ & $1.0 \times 10^{-2} /$ alpha & R35a & $5.0 \times 10^{-4} /$ alpha & $\mathrm{w}$ & 11.665 \\
\hline & & & & Ts & $1 \times$ alpha $^{n}$ \\
\hline
\end{tabular}

Table 5. Values of constants. 